\title{
Existence and Multiplicity of Solutions for a Class of Nonlinear Schrod- inger-KdV Equations
}

\author{
Ping Zhou ${ }^{1, *}$, Chaoqian $\mathrm{Li}^{2}$ and Yanyan $\mathrm{Li}^{1}$ \\ ${ }^{1}$ Institute of mathematics, Wenshan College, ${ }^{2}$ School of mathematics and statistics, Yunnan University
}

\begin{abstract}
In this paper, a class of coupled Riccati equations by using some special solutions of nonlinear coupled Schrodinger-KdV equations of a number of exact analytical group, obtain the precise solution and two groups of new solitary wave solutions of the equations and several general forms. With the help of computer symbolic computation technology, using F- expansion method to obtain exact solutions of the coupled Schrodinger-KdV equations, including the trigonometric function solutions, hyperbolic function solutions and Jacobian elliptic function solutions, the precise solution is widely used in plasma physics. In the past few decades, many scholars on the nonlinear Schrodinger equation and the existence of solutions of multi solution problem is studied, and the nonlinear term of the proposed restriction conditions become more and more weak. This paper firstly studies the ground state spectra with zero point solutions of Schrodinger equation. When the nonlinearity is superlinear, we give a more direct, simple and convenient method.
\end{abstract}

Keywords: Schrodinger-KdV equations, solitary wave solutions, symbolic computation.

\section{INTRODUCTION}

In recent years, with the establishment and development of the nonlinear phenomena of the in-depth study and soliton theory, with many equations have been appear in several fields of fluid mechanics, solid state physics, physics, elementary particles in optical fiber communication and other nonlinear soliton solutions of the evolution.

At present, there are many kinds of methods for solving soliton solutions of nonlinear evolution equations, such as Backlund transform, Jacobi elliptic function method, hyperbolic function method, homogeneous balance method, bilinear method. The demand equations are nonlinear coupling Schrodinger-KdV equations [1-3]:

$$
\left\{\begin{array}{c}
i u_{i}+u_{x x}=u v, \\
v_{i}+\alpha v v_{x}+\beta v_{x x x}=\left(|u|^{2}\right)_{x} .
\end{array}\right.
$$

It is an important model to describe the interaction between long and short wave nonlinear dissipative medium, wherein $U$ represents a real long wave, said V. $\alpha, \beta$ complex shortwave, control parameters, with the physical background can be found in reference 2, 3. In the literature gives equations by Yoshinaga et al $[4,5]$. The improved Hirota method 4 (1) in the five groups of solitary wave solution. Recently, the reference 5, respectively, 6 Improved hyperbolic tangent method 7-10, and obtained some nonlinear equations and the equations of the exact solution by this method (the extended application of these methods in equations (1), with the aid of computer algebraic system Maple and obtained the equations
(1) of the seven groups of solitary wave solutions, these solutions not only contains the Yoshinaga results, more important is to obtain two new sets of regular solitary wave solutions [6].

Fractional order partial differential equation (pde) was an important equation of optics, electromagnetism, quantum mechanics, fluid mechanics, atomic physics, quantum - mechanical system field etc. Many scholars have carried out extensive and in-depth research on it, making the relevant fractal dimension of the problem was well solved. The essential and key of problem in the nature of things were more accurate described by fractional order pde with important the physical background, outstanding research value and broad prospects for the study. At present, research of correlation properties and the application of fractional order pde has become the focal point, in the numerical solution, exact solution and well posedness of the definite solution of problems has made a breakthrough progress $[7,8]$.

This paper discussed the existence of solutions of three types of fractional order nonlinear Schrodinger equation (group). We mainly studied the existence and uniqueness of solution for a class of generalized fractional order nonlinear Schrodinger equations, the cycle boundary value of whole smooth solution for a class of fractional order nonlinear Schrodinger equation with multiple potential, the existence and uniqueness of global smooth solution for a class of the fractional order nonlinear Schrodinger equations with weak damping.

It widely used in plasma physics, such as can be used to describe the Laugmuir wave, electromagnetic wave. The literature [9] equation (1) discussed the integrability of the literature, using [10] elliptic function expansion method is used to solve the equation (1) some exact solutions. With the aid of symbolic computation technology, by using the F- 
expansion method $[11,12]$ to solve the equation (1) exact soliton solutions.

\section{SOLITARY WAVE SOLUTIONS OF THE NON- LINEAR COUPLED SCHRODINGER-KdV EQUA- TIONS}

In order to seek the equations (1) and the traveling wave solution, the introduction of the traveling wave transformation [13]

$$
\left\{\begin{array}{l}
U(x, t)=V(\xi) \exp \left[i \frac{c}{2}(x-V t)\right], \\
V(x, t)=V(\xi)
\end{array}\right.
$$

The $\varepsilon=\mathrm{x}-\mathrm{ct}$, c is an arbitrary non-zero constant (in the transform (2) type

Equations (1) into a system of ordinary differential equations $[14,15]$

$$
\left\{\begin{array}{l}
U^{\prime \prime}+\frac{c}{2}(x-V t) U=U V \\
\beta V^{\prime \prime}+\frac{\alpha}{2} V^{2}-c V=U^{2}-C^{2} .
\end{array}\right.
$$

Where $\mathrm{c}$ is a constant of integration

For the solution of (3) type, consider a class of coupled Riccati equations

$$
\left\{\begin{array}{l}
f^{\prime}(\xi)=-k f(\xi) g(\xi), \\
g^{\prime}(\xi)=k\left(1-g^{2}(\xi)-r f(\xi)\right) .
\end{array}\right.
$$

Where k, R is an arbitrary constant. Equations (4) have a special solution of two group (referred to as the basic solitary wave function).

$$
\begin{gathered}
\left\{\begin{array}{l}
f(\xi)= \pm \frac{1}{\cosh \left[k\left(\xi+\xi_{0}\right)\right]+r} \\
g(\xi)= \pm \frac{\sinh \left[k\left(\xi+\xi_{0}\right)\right]}{\cosh \left[k\left(\xi+\xi_{0}\right)\right]+r}
\end{array}\right. \\
\text { and } \\
\left\{\begin{array}{l}
f(\xi)= \pm \frac{1}{\sinh \left[k\left(\xi+\xi_{0}\right)\right]+r} \\
g(\xi)=\frac{\cosh \left[k\left(\xi+\xi_{0}\right)\right]}{\sinh \left[k\left(\xi+\xi_{0}\right)\right]+r},
\end{array}\right.
\end{gathered}
$$

The $\varepsilon_{0}$ is an arbitrary constant (usually desirable to zero), $\mathrm{K}$ is the wave number, in order to make (5) type solitary wave solutions become regular, say $r>0$ and $r \neq 1$. is easy to see that (5) and (6) type respectively meet:

$$
\begin{aligned}
& g^{2}=1-2 r f+\left(r^{2}-1\right) f^{2} \\
& \text { And } \\
& g^{2}=1-2 r f+\left(r^{2}+1\right) f^{2}
\end{aligned}
$$

Assume that $\mathrm{U}, \mathrm{V}$ can be expressed as a polynomial of $\mathrm{F}$ and basic g solitary wave function, number of respectively $\mathrm{m}, \mathrm{N}$, (3) the balance of linear and nonlinear terms of the highest order derivatives of the number, type in $\mathrm{m}=0,1,2$, $\mathrm{n}=2$, and so can be set:

$\left\{\begin{array}{l}U=a_{0}+a_{1} f+a_{2} f^{2}+b_{1} f+b_{2} f g \\ V=c_{0}+c_{1} f+c_{2} f^{2}+d_{1} f+d_{2} f g\end{array}\right.$

The $\mathrm{c} 2=0$ or $\mathrm{d} 2=0$, taking into account the (7) type, type the maximum number of times in $\mathrm{G}$ to 1 will (9) type substitution (3), by (4), (5) and (7) type, can get on undetermined parameters A0, A1, A2, B1, B2, C0, C1, C2, D1, D2, K, c, $\mathrm{C}, \mathrm{V}$, nonlinear algebraic equations

The elimination method for solving equations (11) in the computer algebra system was on Maple (10), to obtain the equations. By many nontrivial solutions of (9), (5) and (2) type, finally got the equation (1) of the seven groups of regular solitary wave solution, the obtained conclusion the following:

1) when the parameter $\alpha, \beta$, meet $\alpha+6 \beta=0$, equation (1) has two sets of Solutions

$\left\{\begin{array}{l}U_{1}(x, t)=A \operatorname{sech}[k(x-c t)] \exp \left[i \frac{c}{2}(x-V t)\right], \\ V_{1}(x, t)=k^{2}+\frac{c}{2}\left(V-\frac{c}{2}\right)-2 k^{2} \operatorname{sech}^{2}[k(x-c t)],\end{array}\right.$

In it:

$$
A^{2}=k^{2}\left(2 c+4 k^{2} \beta+6 \beta c V-3 \beta c^{2}\right)
$$

The integral constant $\mathrm{C}$ meet:

$C^{2}=\frac{1}{16}\left(4 k^{2}-c^{2}+2 c V\right)\left(12 k^{2} \beta-3 \beta c^{2}+6 \beta c V+4 c\right)$,

$\mathrm{K}, \mathrm{C}, \mathrm{V}$ is an arbitrary constant. Obviously, if the 0 taken as zero, we must make it a factor is zero, for example $4 k^{2}-c^{2}+2 c V=0$, at this time, the solution of (10) type:

$$
\left\{\begin{array}{l}
U_{2}(x, t)=A \tanh [k(x-c t)] \exp \left[i \frac{c}{2}(x-V t)\right], \\
V_{2}(x, t)=\frac{c}{2}\left(V-\frac{c}{2}\right)-2 k^{2} \operatorname{sech}^{2}[k(x-c t)],
\end{array}\right.
$$

In it:

$$
A^{2}=k^{2}\left(-2 c-6 \beta c V+3 \beta c^{2}+8 k^{2} \beta\right)
$$

$\mathrm{k}, \mathrm{c}, \mathrm{V}$ is an arbitrary constant, the integral constant $\mathrm{C}$ meet:

$$
\begin{aligned}
C^{2}= & -\frac{1}{16}\left(32 c k^{2}+96 \beta k^{2} c V-48 \beta k^{2} c^{2}-128 \beta k^{4}\right. \\
& \left.-12 \beta c^{2} V^{2}+12 \beta c^{3} V-3 \beta c^{4}-8 c^{2} V+4 c^{3}\right) \\
C^{2}= & -\frac{1}{16}\left(32 c k^{2}+96 \beta k^{2} c V-48 \beta k^{2} c^{2}-128 \beta k^{4}\right. \\
& \left.-12 \beta c^{2} V^{2}+12 \beta c^{3} V-3 \beta c^{4}-8 c^{2} V+4 c^{3}\right) .
\end{aligned}
$$


When we solution the equation (12), we let $V=\frac{c}{2}$, then $C^{2}=A^{2}=2 k^{2}\left(-c+4 k^{2} \beta\right)$, the equation (12) is the equation 2.9 in document [16-18].

2) If parameters $\alpha, \beta$ meets $\alpha+2 \beta>0$, the equations (1) have two solutions:

$\left\{\begin{array}{l}U_{3}(x, t)=A \sec h^{2}[k(x-c t)] \exp \left[i \frac{c}{2}(x-V t)\right], \\ V_{3}(x, t)=\frac{c-4 \beta k^{2}}{\alpha}-6 k^{2} \sec h^{2}[k(x-c t)],\end{array}\right.$

Among them

$$
A^{2}=18 k^{4}(\alpha+2 \beta), k^{2}=\frac{c(c \alpha+4-2 V \alpha)}{16(\alpha+\beta)},
$$

The constant of integration $\mathrm{C}$ is to meet

$$
C^{2}=\frac{c^{2}-16 \beta^{2} k^{4}}{2 \alpha}
$$

$c, V$ is random constant,

$$
c(c \alpha+4-2 V \alpha)(\alpha+\beta)>0 \text {. }
$$

In the solution of equation (14), Take $V=\frac{c}{2}-\frac{2}{\beta}$, then $k^{2}=\frac{c}{4 \beta}, C=0$, the solution of equation (14) degenerate to the equation 2.8 in document [4].

$$
\left\{\begin{aligned}
U_{4}(x, t)= & A\left\{1-\frac{3}{2} \sec h^{2}[k(x-c t)]\right\} \\
& \times \exp \left[i \frac{c}{2}(x-V t)\right], \\
V_{4}(x, t)= & \frac{c}{2}\left(V-\frac{c}{2}\right)-6 k^{2} \sec h^{2}[k(x-c t)],
\end{aligned}\right.
$$

Among them

$$
A^{2}=8 k^{4}(\alpha+2 \beta), k^{2}=-\frac{c(c \alpha+4-12 V \alpha)}{16(\alpha+\beta)},
$$

The constant of integration $\mathrm{C}$ is to meet

$$
C^{2}=\frac{c^{2}-16 \beta^{2} k^{4}}{2 \alpha},
$$

$\mathrm{c}, \mathrm{V}$ is random constant,

$c(c \alpha+4-2 V \alpha)(\alpha+\beta)<0$. In the solution of equation (15), Take $V=\frac{c}{2}$, then the solution of equation (15) degenerate to the equation 2.10 in document [19].
3) If parameters $\alpha, \beta$ meets $\alpha+2 \beta<0$, the equations (1) have one solution:

$$
\left\{\begin{aligned}
U_{5}(x, t)= & A \tan h[k(x-c t)] \sec h[k(x-c t)] \\
& \times \exp \left[i \frac{c}{2}(x-V t)\right] \\
V_{5}(x, t)= & k^{2}+\frac{c}{2}\left(V-\frac{c}{2}\right) \\
& -6 k^{2} \sec ^{2}[k(x-c t)]
\end{aligned}\right.
$$

Among them

$$
A^{2}=-18 k^{4}(\alpha+2 \beta), k^{2}=-\frac{c(c \alpha+4-2 V \alpha)}{8(\alpha+\beta)}, \quad \text { The }
$$
constant of integration $\mathrm{C}$ is to meet

$$
C^{2}=-\frac{1}{32}\left(c^{2}-4 k^{2}-2 c V\right)\left(\alpha c^{2}-2 \alpha c V+8 c-4 \alpha k^{2}\right),
$$

c, $\mathrm{V}$ is random constant,

$c(c \alpha+4-2 V \alpha)(\alpha+\beta)<0$. If we take the factor of $C^{2}: c^{2}-4 k^{2}-2 c V=0$, then we can get the conclusion

$V=\frac{c}{2}+\frac{2}{3 \alpha+2 \beta}$, which is just the condition has been given in document [20], at the time, the solution of equation (16) degenerate to the equation 2.7 in document [4].

4) If parameters $\alpha, \beta$ meets $3 \alpha+4 \beta=0, \beta>0$, the equations (1) have one solution:

$$
\left\{\begin{aligned}
U_{6}(x, t)= & \pm 5 \sqrt{6 \beta} k^{2} \frac{\sqrt{7} \cosh (x-c t)+2 \sqrt{2}}{(\sqrt{7} \cosh (x-c t)+\sqrt{2})^{2}} \\
& \times \exp \left[i \frac{c}{2}(x-V t)\right], \\
V_{4}(x, t)= & k^{2}+\frac{1}{2} c V-\frac{1}{4} c^{2} \\
& -30 k^{2} \frac{1}{(\sqrt{7} \cosh (x-c t)+\sqrt{2})^{2}},
\end{aligned}\right.
$$

Among them

$$
V=\frac{1}{2} \frac{23 \beta k^{2}+\beta c^{2}-3 c}{\beta c},
$$

$k$ and $c$ arerandom constant, the constant of integration $\mathrm{C}$ is to meet

$$
C= \pm \frac{1}{4} \frac{\sqrt{-6 \beta\left(c^{2}-81 \beta^{2} k^{4}\right)}}{\beta} .
$$

5) If parameters $\alpha, \beta$ meets $3 \alpha+4 \beta=0, \beta<0$, the equations (1) have one solution: 


$$
\left\{\begin{aligned}
U_{7}(x, t)= & \pm 3 \sqrt{-10 \beta} k^{2} \sinh (x-c t)(\sqrt{5} \cosh (x \\
& -c t)+2 \sqrt{2})(\sqrt{5} \cosh (x-c t)+\sqrt{2})^{-2} \\
& \times \exp \left[i \frac{c}{2}(x-V t)\right] \\
V_{7}(x, t)= & \frac{c}{2}\left(V-\frac{c}{2}\right) \\
& -18 k^{2} \frac{1}{(\sqrt{5} \cosh (x-c t)+\sqrt{2})^{2}}
\end{aligned}\right.
$$

Among them

$V=\frac{c}{2}+\frac{39 k^{2}}{2 c}-\frac{3}{2 \beta}, \mathrm{k}$ and $\mathrm{c}$ are random constant, the constant of integration $\mathrm{C}$ is to meet $C= \pm \frac{1}{4} \frac{\sqrt{-6 \beta\left(c^{2}-121 \beta^{2} k^{4}\right)}}{\beta}$. The solution of equations(17) and (18) is the newly acquiredin this paper.

\section{EXISTENCE OF SCHRODINGER-KdV EQUA- TIONS}

Theorem: Let $\alpha>\frac{n}{2}$, if $\rho$ is an even number, when $\beta, \gamma>0$, set $\rho, \mu>0$, when $\beta, \gamma<0$, set $0<\rho, \mu<\frac{4 \alpha}{n}$, if $\rho$ is not an even number, when $\beta, \gamma>0$, set $\rho, \mu>2|\alpha|+1$, when $\beta, \gamma<0$, set $2|\alpha|+1<\rho, \mu<\frac{4 \alpha}{n}$, for all $u_{0} \in H_{p e r}^{4 \alpha}(\Omega)$, then there was a unique whole smooth solution $u(x, t)$ for the problem of (12) (16) making

$$
u \in L^{\infty}\left(0, \mathrm{~T} ; H_{p e r}^{4 \alpha}(\Omega)\right), u_{t} \in L^{\infty}\left(0, \mathrm{~T} ; H_{p e r}^{2 \alpha}(\Omega)\right) .
$$

For integer order nonlinear Schrodinger equation (group) has been the result of system. In the 80's Bailing Guo fellow in-depth studied this kind of equation (Group), and in the paper [11], the authors studied the existence of whole solutions of the initial value problem for a class of integer order nonlinear Schrodinger equations, Where $q(s)(>0)$ was the real valued function on $[0,+\infty]$, and $k_{j}(x, t), f_{j}(x, t)$ was known to complex valued functions.

$$
\begin{aligned}
& u_{1 t}-i u_{1 x x}+B q\left(\left|u_{1}\right|^{2}+\left|u_{2}\right|^{2}\right) u_{1}+k_{1}(x, t) u_{1}=f_{1}(x, t), \\
& u_{2 t}-i u_{2 x x}+B q\left(\left|u_{1}\right|^{2}+\left|u_{2}\right|^{2}\right) u_{2}+k_{2}(x, t) u_{2}=f_{1}(x, t), \\
& \left.u_{1}\right|_{t=0}=u_{0}^{1}(x),\left.\quad u_{2}\right|_{t=0}=u_{0}^{2}(x), \quad-\infty<x<\infty
\end{aligned}
$$

\section{INFINITELY MANY SOLUTIONS OF SCHROD- INGER-KdV EQUATIONS}

In this chapter we consider the bounded domains is a special class of Semilinear Schrodinger equation, in previous work are required for the nonlinear term is non negative, while the nonlinear in we give conditions is the middle term changes sign, in a more general super two conditions, we get the problem of infinite a solution.

\subsection{The Main Results}

For the problem, we give the following hypothesis:

(G1) $f \in C(\Omega \times R, R)$, and there exists constant

$c_{1}>0$ and $p \in\left(2,2^{*}\right)$,

making

$|f(x, t)| \leq c_{1}\left(1+|t|^{p-1}\right), \quad \forall(x, t) \in \Omega \times R ;$

(G2) $\lim _{|t| \rightarrow \infty} \frac{|F(x, t)|}{|t|^{2}}=\infty, a, e, x \in \Omega$,

and there exists constant $r_{0} \geq 0$,

making $F(x, t) \geq 0, \quad \forall(x, t) \in \Omega \times R,|t| \geq r_{0}$;

(G3) $F(x, t)=\frac{1}{2} t f(x, t)-F(x, t)$, and there exists $c_{0}>0$ and $k>N / 2$, making

$0 \leq|F(x, t)|^{k} \leq c_{0}|t|^{2 k} F(x, t), \forall(x, t) \in \Omega \times R,|t| \geq r_{0} ;$

(G4) $f(x,-t)=-f(x, t), \forall(x, t) \in \Omega \times R$;

(G5) There exists $\mu>2$ and $\lambda>0$, making

$$
\mu F(x, t) \leq t f(x, t)+\lambda t^{2}, \forall(x, t) \in \Omega \times R .
$$

In the following discussion, $\mid \Lambda_{\mid}$was defined as the absolute value of $\Lambda,|\Lambda|^{1 / 2}$ as the square root of

$\mid \Lambda_{\mid}$. Its domain of definition was $D\left(|\Lambda|^{1 / 2}\right)$, then $E=D\left(|\Lambda|^{1 / 2}\right)=H_{0}^{1}(\Omega)$. Let $\theta$ is a positive constant, meet $\mu_{1}>-\theta$, where $\mu_{1}$ is the minimum feature value of $\Lambda$, then $\Lambda+\theta I \geq 0$.

We introduced the following inner product on $\mathrm{E}$ :

$(u, v)=\left((\Lambda+\theta I)^{1 / 2} u,(\Lambda+\theta I)^{1 / 2} v\right)_{2}+(u, v)_{2}$

and norm $\|u\|=(u, u)^{1 / 2}$, where $(\cdot, \cdot)_{2}$ was norm of $L_{2}(\Omega)$. Then the norm $\|\cdot\|$ was equivalent to the usual norm $\|\cdot\|_{H^{1}(\Omega)}$. Let

$V(x)=a(x)+\theta+1, g(x, u)=f(x, u)+(\theta+1) u$

easy to verify, if $f(x, u)$ meet the conditions (G1) (G5), then $g(x, u)$ also meet $(\mathrm{G} 1) \sim(\mathrm{G} 5)$. Therefore, we have the following lemma.

This paper firstly studies on the ground state space with zero spectral point solution. When the nonlinearity is superlinear, we give a more direct ground state solution, simple method, in the absence of strict monotonicity condition, obtain the existence of solutions of Nehari - Pankov type of 
ground state. When the nonlinear term is asymptotically linear time, we first study the existence of nontrivial weak solution of the problem, and on the assumption that the condition is relatively loose, proved the least energy solution and $\mathrm{Ne}-$ hari - Pankov of the ground state solutions existence.

In the last chapter of the paper, we also consider the solution bounded domain problems, in a more general super two conditions, we prove that there are infinitely many solutions for, in previous work are required for the nonlinear term is non negative, while the nonlinear in our condition is you can change the number, thus greatly improve previous results. In addition, we find there are many problems can be further research, can carry out the work from the following aspects:

First, this paper only considers the problem of the existence of the Schrodinger spectrum with zero point equation solution, can further consider the multiplicity of solutions for problems. Notice, established in literature generalized surround theorem improves linking theorem in the past, we discuss in this paper is very useful to the problem, can similarly create a critical point on the multiple solution theorem, this is also what we have to do the work.

Second, in discussing the solution of Schrodinger equation of the ground state zero point spectrum with potential requirements, is periodic, the potential is asymptotically periodic case, whether can also discuss the existence of ground state solutions, this is also the question.

\section{CONCLUSION}

From the conclusion of this paper can be seen, we obtain the exact solution of the Schrodinger-KdV equations of the form [1] is more general than the literature, not only that, we also had two group new. In addition, if the use of the solution to (6) type and relation type (8), similarly also can obtain equations (1) solitary wave solutions of the singular form.

The main results of this paper consists of three parts. First, studied the well posedness of solutions for a class of generalized fractional order nonlinear Schrodinger equations. Let a priori estimates was a solution with a large number of application inequalities of Gagliardo-Nirenberg, Young's and Gronwnll. In order to more accurately estimate the boundedness of solutions, the nonlinearity of " $g\left(\left|u_{1}\right|^{2}+\left|u_{2}\right|^{2}\right)$ " and its derivative, the two derivative were limited, then the existence of solution was got by Facdo-Galerkin method.

Finally, the existence and uniqueness of solutions was proved.

Second, the cycle boundary value of whole smooth solution for fractional order nonlinear Schrodinger equation with multiple potential was sconsidered.

Third, the existence and uniqueness of global smooth solution for the fractional order nonlinear Schrodinger equations with weak damping was studied.

\section{CONFLICT OF INTEREST}

The authors confirm that this article content has no conflict of interest.

\section{ACKNOWLEDGEMENTS}

The research work was supported by Yunnan province science and Technology Department of applied basic research on Youth Project under Grant No. 2013FD052 and the project of the key discipline construction of Wenshan University under Grant No. 12WSXK01.

\section{REFERENCES}

[1] C. J. Batkam, and F. Colin, "On multiple solutions of a semilinear SchrSdinger equation with periodic potential," Nonlinear Analysis, vol. 84, pp. 2677-2694, 2013.

[2] T. Bartsch, and Y. Ding, "On a nonlinear Schrodinger equation with periodic potential,” Math. Ann., vol. 313, pp. 15-37, 1999.

[3] T. Bartsch, and Y. Ding, "Deformation theorems on non-metrizable vector spaces and applications to critical point theory," Math. Nachrichten, vol. 279, pp. 1267-1288, 2006.

[4] B. Buffoni, L. Jeanjean, and C.A. Stuart, "Existence of nontrivial solutions to a strongly indefinite semilinear equation," Proc. Amer. Math. Soc., vol. 119, pp. 179-186, 1993.

[5] T. Bartsch, A. Pankov, and Z.Q. Wang, "Nonlinear SchrSdinger equations with steep potential well," Commun. Contemp. Math., vol. 3, no. 4, pp. 549-569, 2011.

[6] T. Bartsch, and Z. Q. Wang, "Existence and multiplicity results for some superlinear elliptic problems on MN, Comm," Partial Differential Equations, vol. 20, pp. 1725-1741, 1995.

[7] T. Bartsch, and M. Willem, "Infinitely many solutions of a symmetric Drichlet problem," Nonlinear Anal., vol. 20, pp. 1205-1216, 1993.

[8] J. Byeon, and Z.Q. Wang, "Standing waves with a critical frequency for nonlinear Schrödinger equations II," Calc. Var. Partial Differential Equations, vol. 18, pp. 207-219, 2003.

[9] D.G. Costa, "Variational problems which are nonquadratic at infinity," in: H.Brezis(Ed.), Morse Theorem, Minimax Theory and Their Applications to Nonlinear Differential Equuations, International Press, Somerville, MA, 2003.

[10] V. C. Zelati, and P.H. Rabinowitz, "Homoclinic type solutions for a semilinear elliptic PDE on RN, Comm.," Pure Appl. Math. XIV, pp. 1217-1269, 1992.

[11] K. W. Chow, K. K. Y. Wong, and K. Lam, "Modulation instabilities in a system of four coupled nonlinear Schr" odinger equations," Physics Letters A, vol. 372, no. 25, pp. 4596-4600, 2008.

[12] K. W. Chow, "Periodic waves for a system of coupled, higher order nonlinear Schr"odinger equations with third order dispersion," Physics Letter A, vol. 308, no. 5-6, pp. 426-431, 2003.

[13] N. Laskin, "Fractional quantum mechanics and L'evy integrals," Physics Letters A, vol. 268, no. 4-6, pp. 298-305, 2000.

[14] N. Laskin, "Fractional quantum mechanics," Phys. Rev. E, vol. 62, no. 3, pp. 3135-3145, 2000.

[15] J. Wang, Y. Zhou, and W. Wei, "Fractional Schr"odinger equations with potential and optimal controls," Nonlinear Analysis: Real World Applications, vol. 13, no. 6, pp. 2755-2766, 2012.

[16] X. Guo, and M. Xu, "Some physical applications of fractional Schro"dinger equation," J. Math. Phys, vol. 47, no. 8, pp. 082104082104-9, 2006.

[17] S. Wang, and M. Xu, "Generalized fractional Schr" odinger equation with space space time fractional derivatives," J. Math. Phys, vol. 48, no. 4, pp. 043502-043502-10, 2007.

[18] C. C. Bradley, C. A. Sackett, and R. G. Hulet, "Bose-Einstein condensation of Lithium: observation of limited condensate number," Phys Rev Lett, vol. 78, no. 6, pp. 985-989, 1997. 
[19] B. M. Caradoc-Davies, R. J. Ballagh, and K. Burnett, "Coherent dynamics of vortex formation in trapped Bose-Einstein condensates," Phys Rev Lett, vol. 83, no. 5, pp. 895-898, 1999.
[20] F. Dalfovo, S. Giorgini, L. P. Pitaevskii, and S. Stringari, "Theory of Bose-Einstein condensation in trapped gases," Reviews of Modern Physics, vol. 71, no. 3, pp. 463-512, 1999.

Received: June 10, 2015

Revised: July 29, 2015

Accepted: August 15, 2015

(C) Zhou et al.; Licensee Bentham Open.

This is an open access article licensed under the terms of the (https://creativecommons.org/licenses/by/4.0/legalcode), which permits unrestricted, noncommercial use, distribution and reproduction in any medium, provided the work is properly cited. 